\title{
ARTICLE OPEN Empirical comparison of reduced representation bisulfite sequencing and Infinium BeadChip reproducibility and coverage of DNA methylation in humans
}

Juan J. Carmona ${ }^{1,2,3}$, William P. Accomando Jr. ${ }^{4,5}$, Alexandra M. Binder ${ }^{4,5}$, John N. Hutchinson ${ }^{6}$, Lorena Pantano ${ }^{6}$, Benedetta Izzi ${ }^{7}$, Allan C. Just ${ }^{1}$, Xihong Lin $^{6}$, Joel Schwartz ${ }^{1}$, Pantel S. Vokonas ${ }^{8}$, Sami S. Amr ${ }^{9,10}{ }^{\text {, Andrea A. Baccarelli }}{ }^{1,4}$ and Karin B. Michels ${ }^{4,5}$

We empirically examined the strengths and weaknesses of two human genome-wide DNA methylation platforms: rapid multiplexed reduced representation bisulfite sequencing and Illumina's Infinium BeadChip. Rapid multiplexed reduced representation bisulfite sequencing required less input DNA, offered more flexibility in coverage, and interrogated more CpG loci at a higher regional density. The Infinium covered slightly more protein coding, cancer-associated and mitochondrial-related genes, both platforms covered all known imprinting clusters, and rapid multiplexed reduced representation bisulfite sequencing covered more microRNA genes than the HumanMethylation450, but fewer than the MethylationEPIC. Rapid multiplexed reduced representation bisulfite sequencing did not always interrogate exactly the same CpG loci, but genomic tiling improved overlap between different libraries. Reproducibility of rapid multiplexed reduced representation bisulfite sequencing and concordance between the platforms increased with CpG density. Only rapid multiplexed reduced representation bisulfite sequencing could genotype samples and measure allele-specific methylation, and we confirmed that Infinium measurements are influenced by nearby single-nucleotide polymorphisms. The respective strengths and weaknesses of these two genome-wide DNA methylation platforms need to be considered when conducting human epigenetic studies.

npj Genomic Medicine (2017)2:13; doi:10.1038/s41525-017-0012-9

\section{INTRODUCTION}

Epigenetics is the study of mitotically and/or meiotically heritable gene regulation that is not due to changes in the primary sequence of DNA nucleotides. ${ }^{1}$ DNA methylation (DNAm) at cytosine residues in cytosine-guanine $(\mathrm{CPG})$ dinucleotides is one of the most studied epigenetic marks; it is relatively easy to measure, critical to the maintenance of cellular identity, ${ }^{2,3}$ and related to chromatin conformation and transcriptional programming. ${ }^{3-6}$ CpG loci are statistically underrepresented in mammalian genomes, but they are often concentrated in regions known as CpG "islands", and $\sim 60 \%$ of known human gene promoters contain CpG islands. ${ }^{7}$ Genomic regions 2000 base-pairs (2 kb) to each side of a CpG island are CpG "shores," with CpG "shelves" extending $2 \mathrm{~kb}$ beyond $\mathrm{CpG}$ shores, with the rest of the genome termed "open sea". These four contexts form the CpG "resort", and the concentration of CpG loci decreases from islands to the open sea. ${ }^{8}$ The context of interest may depend on the research question. For example, since DNAm within promoter CpG islands exhibits patterns established during cellular differentiation and passed down through cell lineages, ${ }^{9}$ scientists investigating cell- lineage-specific gene regulation and/or identifying epigenetic biomarkers of cell and tissue types may focus on promoter CpG islands. ${ }^{10-13}$ On the other hand, since DNAm in CpG shores and shelves is more responsive to external factors, ${ }^{14-16}$ scientists investigating environmental programming of the genome via DNAm and/or trying to determine whether DNAm mediates known associations between exposures and diseases may focus on CpG shores and shelves.

Approaches that measure DNAm are continually being developed and refined. Many probe- and sequencing-based DNAm quantification approaches take advantage of sodium bisulfite treatment, which converts unmethylated cytosines to uracil (becoming thymine after PCR amplification) without changing methylated cytosines, allowing quantification of DNAm via estimation of cytosine-to-thymine at known CpG loci. ${ }^{17}$ Probebased detection employs site-specific probes that hybridize onto bisulfite-converted DNA at target CpG loci, resulting in fluorescent signals. Infinium BeadChip arrays from Illumina (San Diego, CA), including the HumanMethyation450 (450K), and MethylationEPIC (850K), measure DNAm at pre-defined CpG loci with a generally

\footnotetext{
${ }^{1}$ Department of Environmental Health, Harvard T.H. Chan School of Public Health, Boston, MA 02115, USA; ${ }^{2}$ Program in Quantitative Genomics, Harvard T.H. Chan School of Public Health, Boston, MA 02115, USA; ${ }^{3}$ Center for Bioethics, Harvard Medical School, Boston, MA 02115, USA; ${ }^{4}$ Department of Epidemiology, Harvard T.H. Chan School of Public Health, Boston, MA 02115, USA; ${ }^{5}$ Obstetrics and Gynecology Epidemiology Center, Department of Obstetrics, Gynecology and Reproductive Biology, Brigham and Women's Hospital, Harvard Medical School, Boston, MA 02115, USA; ${ }^{6}$ Department of Biostatistics, Harvard T. H. Chan School of Public Health, Boston, MA 02115, USA; ${ }^{7}$ Institute for Prevention and Cancer Epidemiology, Freiburg Medical Center, University of Freiburg, Freiburg, Germany; ${ }^{8}$ Veterans Affairs Normative Aging Study, Veterans Affairs Boston Healthcare System, Department of Medicine, Boston University School of Medicine, Boston, MA 02118, USA; ${ }^{2}$ Department of Pathology, Harvard Medical School, Brigham and Women's Hospital, Boston, MA 02115, USA and ${ }^{10}$ Translational Genomics Core, Partners Healthcare Personalized Medicine, Cambridge, MA 02139, USA

Correspondence: Karin B. Michels (k.michels@ucla.edu)

Juan J. Carmona, William P. Accomando and Alexandra M. Binder contributed equally to this work.

Andrea A. Baccarelli and Karin B. Michels jointly supervised this work.
}

Received: 26 September 2016 Revised: 4 January 2017 Accepted: 12 January 2017

Published online: 19 April 2017 


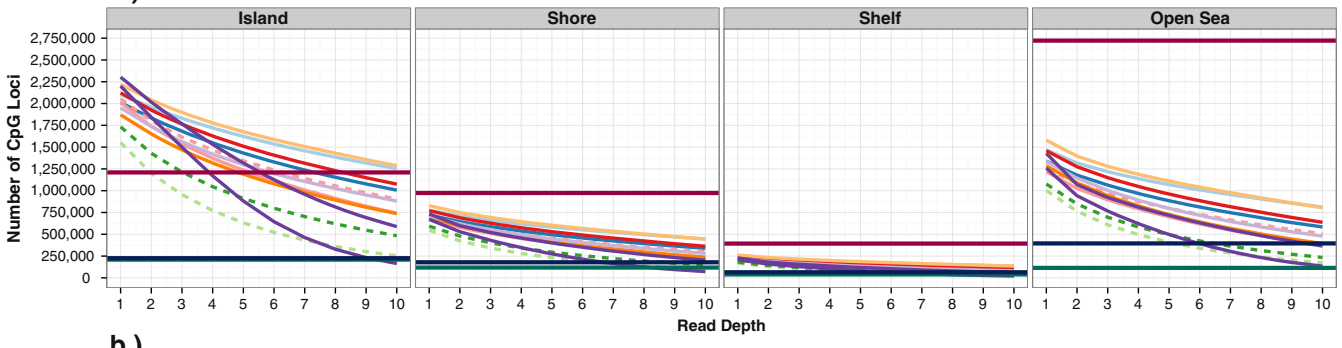

b.)

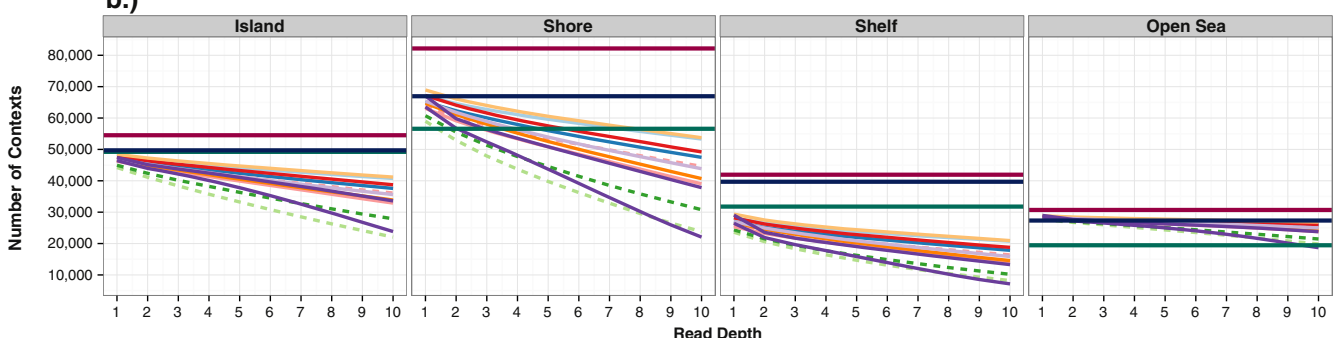

c.)

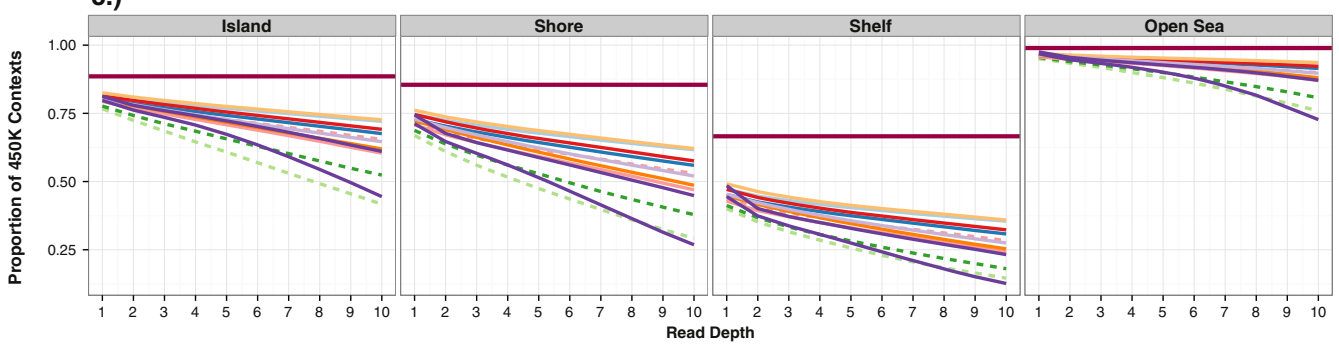

d.)

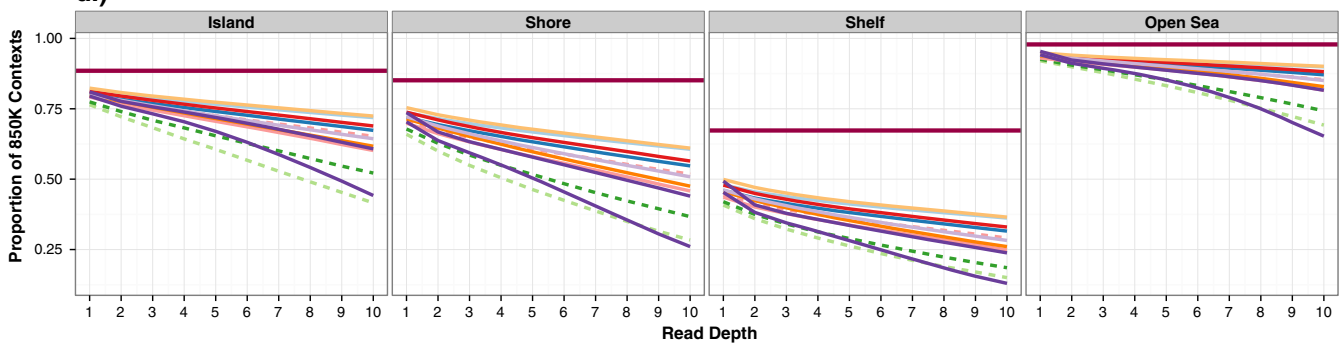

e.)

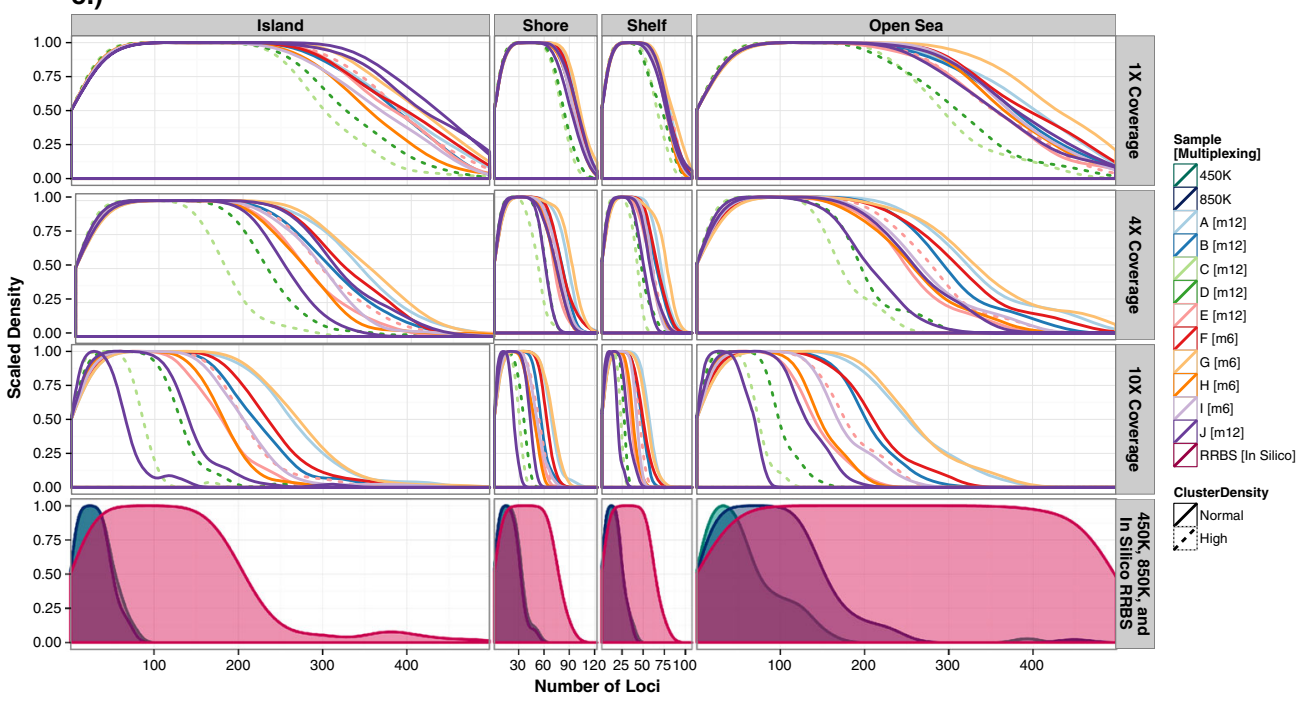


Fig. 1 Genomic coverage of 12 rmRRBS libraries at different read depths stratified by CpG resort context. a The total number of CpG loci covered, $\mathbf{b}$ the number of discrete contexts in which at least one CpG locus is covered, $\mathbf{c}$ the proportion of exactly the same contexts from the Infinium HumanMethyaltion450 (450K) array for which at least once CpG locus was covered, and $\mathbf{d}$ the proportion of exactly the same contexts from the Infinium MethyaltionEPIC (850K) array for which at least once CpG locus is covered are plotted from $\geq 1 \times$ to $\geq 10 \times$ reads. In panels (a)-(d), the dark red horizontal lines indicate predicted RRBS genomic coverage, bioinformatically determined by performing RRBS in silico. In panels (a) and (b), the dark green horizontal lines indicate Infinium 450K genomic coverage, and the dark blue horizontal lines indicate Infinium $850 \mathrm{~K}$ genomic coverage. (e) The distribution of the number of CpG loci measured in each discrete region that was covered is stratified in columns by type of CpG context and in rows by rmRRBS read depth, with CpG density distributions for in silico RRBS, the Infinium $450 \mathrm{~K}$, and the Infinium $850 \mathrm{~K}$ plotted together in the fourth row. The peak of the density is the mode, and indicates most common number of CpG loci measured in each region. In all panels, unique individuals A through $\mathrm{J}$ appear as different colored lines where solid lines indicate normal cluster density and dotted lines indicated high cluster density

high level of reproducibility and reliability, ${ }^{8,18-20}$ but share some limitations. The required input DNA of $500 \mathrm{ng}-1 \mu \mathrm{g}$ precludes the use of Infinium arrays for scarce/precious samples, such as microdissected cancer biopsies. The invariable (and limited) set of CpG loci on Infinium arrays were designed to capture RefSeq genes and promoter $\mathrm{CpG}$ islands, excluding other regions of biologically meaningful variation. Customizable DNAm array options, such as Illumina's VeraCode GoldenGate Methylation Assay use older technology ${ }^{21}$ and only examine a small number of CpG loci (384 loci per array), which are restricted by probe chemistry. Newer Infinium arrays also have issues with dye-biases, different probe chemistries and positional effects that are known to influence results and must be corrected during data processing. ${ }^{22-24}$ Infinium $450 \mathrm{~K}$ probe cross-reactivity and ambiguous mapping to multiple locations in the human genome affects $\sim 140,000$ out of 485,000 probes ( $29 \%$ of the array), potentially reducing the number of usable probes to $\sim 345,000,25,26$ an issue that persists with the newly released $850 \mathrm{~K}$, which includes $>90 \%$ of the $450 \mathrm{~K}$ probes.

Sequencing-based approaches for measuring DNAm across the human genome have rapidly evolved over the last decade. Wholegenome bisulfite sequencing (WGBS) requires a very large amount of DNA, often $3 \mu \mathrm{g}$, to generate a large amount of data that is expensive to store, much of which is not useful due to a lack of variability and/or overlap between samples. ${ }^{27-29}$ To overcome these limitations, reduced representation bisulfite sequencing (RRBS) was developed, requiring less DNA (10-200 ng) and able to generate more meaningful data than WGBS. ${ }^{30} \mathrm{~A}$ modified form of generalized RRBS, multiplexed RRBS (mRRBS) improved feasibility for large studies by allowing multiple libraries per sequencing lane ${ }^{29}$ and others have further modified mRRBS for particular applications. ${ }^{31,32}$ We refined mRRBS to allow for faster and more efficient throughput, thus creating a "rapid multiplexed" RRBS (rmRRBS) platform for the quantification of genome-wide DNAm. Briefly, genomic DNA (gDNA) is digested with Mspl restriction enzyme, which targets CpG-rich areas, to generate a library of fragments that each contains at least two $\mathrm{CpG}$ loci. After ligating indexed oligonucleotide adapters to these fragments and performing size selection using magnetic beads, rmRRBS libraries are pooled, treated with sodium bisulfite, PCR amplified, cleaned up, and then subjected to next-generation sequencing (NGS). The sequenced reads are then aligned to a reference genome and stacked to yield "read depth," i.e., the number of reads per region (e.g., $1 \times, 2 \times$, etc.). Unlike probe-based approaches, sequencingbased approaches like rmRRBS are able to measure singlenucleotide polymorphisms (SNPs) and quantify allele specific DNAm (ASM). ${ }^{3-35}$ SNPs can impact DNAm estimates by altering the primary sequence of nucleotides to eliminate (or add) a CpG locus, and by influencing DNAm at nearby CpG loci. ${ }^{36}$ ASM is of particular interest to studies of human imprinting, a phenomenon where one parental allele is expressed in a parent-of-originspecific manner, while the other is silenced. For example, the 11 p15 chromosomal region contains a contiguous multigene imprinting cluster including $\mathrm{H} 19$, a long non-coding RNA gene that is only expressed by the hypomethylated maternal allele, while the hypermethylated paternal allele is silenced. ${ }^{37}$ Loss of imprinting in $11 \mathrm{p} 15$ is associated with childhood growth disorders and cancer, such as Beckwith-Wiedemann syndrome and Wilms tumor, $^{38}$ as well as adult cancers. ${ }^{39}$

This work is an in-depth examination of rmRRBS genomic coverage and precision for a range of human DNA input quantities. We also explore analytic approaches to RRBS data, including genomic tiling, SNP detection, and ASM quantification. Our goal is to inform researchers who are considering probebased and/or sequencing-based genome-wide DNAm platforms for epigenetic investigations from basic science to epidemiologic studies.

\section{RESULTS}

\section{Genomic coverage}

We constructed and sequenced 86 rmRRBS libraries using human peripheral blood gDNA obtained from 10 adult males, labeled A-J (Supplementary Table S1), which we also evaluated via the Infinium 450K. We performed RRBS in silico to indicate expected RRBS coverage, and used Illumina's manifest files to determine expected $450 \mathrm{~K}$ and $850 \mathrm{~K}$ coverage. We first selected 12 of the rmRRBS libraries-including two technical replicates-to examine in detail. Reflecting the rmRRBS enrichment protocol, genomic coverage varied by genetic element (Figs. 1 and 2; Supplementary Table S2). The number of reads at specific CpG loci for each of the 12 libraries can be viewed in the University of California Santa Cruz Genome Browser (see Supplemental Materials). Stratifying by CpG resort context, all 12 rmRRBS libraries covered from hundreds to over a million more CpG loci than the Infinium arrays at $\geq 4 \times$, and five to ten libraries covered from hundreds to over a million more CpG loci than the Infinium arrays at $\geq 10 \times$ (Fig. 1a). This trend was recapitulated when we stratified by four categories of human genes: protein-coding genes, cancer-associated genes, nuclearencoded genes related to mitochondrial function, and microRNA (miRNA) genes (Fig. 2a). With the exception of CpG islands and miRNA genes, our rmRRBS libraries did not perform as well as predicted by performing RRBS in silico.

Five libraries covered a greater diversity of discrete $\mathrm{CpG}$ shores than the $450 \mathrm{~K}$ at $\geq 4 \times$, eleven libraries covered more open sea regions than the $450 \mathrm{~K}$ at $\geq 10 \times$, but none covered as many $\mathrm{CpG}$ islands and shelves as the $450 \mathrm{~K}$, and the $850 \mathrm{~K}$ covers at least as many of all four contexts as the rmRRBS libraries (Fig. 1b). At $\geq 4 \times$, a majority of the rmRRBS libraries measured at least one CpG locus in $\sim 75 \%$ and $\sim 65 \%$ of the exact same CpG islands and shores, respectively, captured on the Infinium arrays, but only about a third of the exact same $C p G$ shelves (Fig. 1c-d). Even at $\geq 10 \times$, nine rmRRBS libraries covered a larger number of microRNA genes than the $450 \mathrm{~K}$ and a comparable absolute number of cancer-associated genes, protein-coding genes, and nuclear-encoded genes with mitochondrial function to both the $450 \mathrm{~K}$ and $850 \mathrm{~K}$ (Fig. 2b). Moreover, overlap between the rmRRBS libraries at $\geq 10 \times$ and the Infinium arrays ranged from $83 \%$ to $93 \%$ for protein-coding genes, 93-98\% for cancer-associated genes, $80-96 \%$ for mitochondrial- 
COPJ Comparison of RRBS and Infinium DNA methylation
$\mathrm{JJ}$ Carmona et al
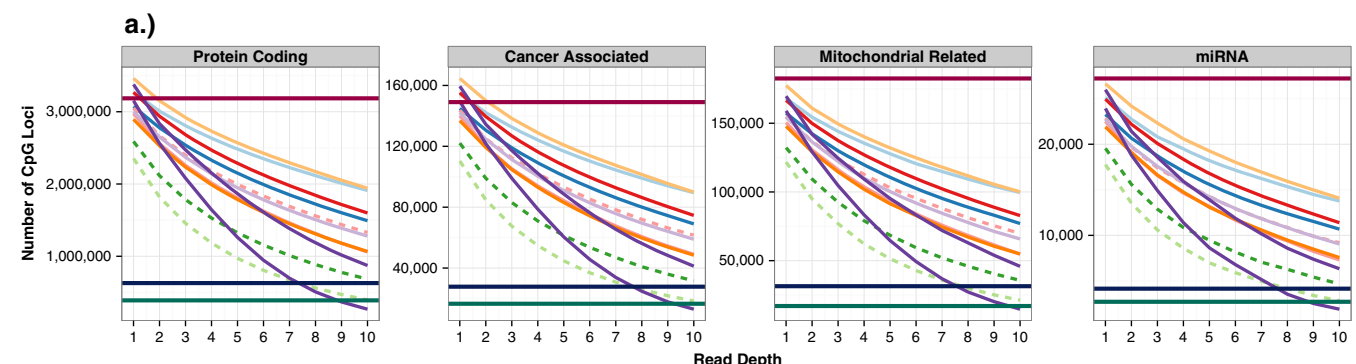

b.)
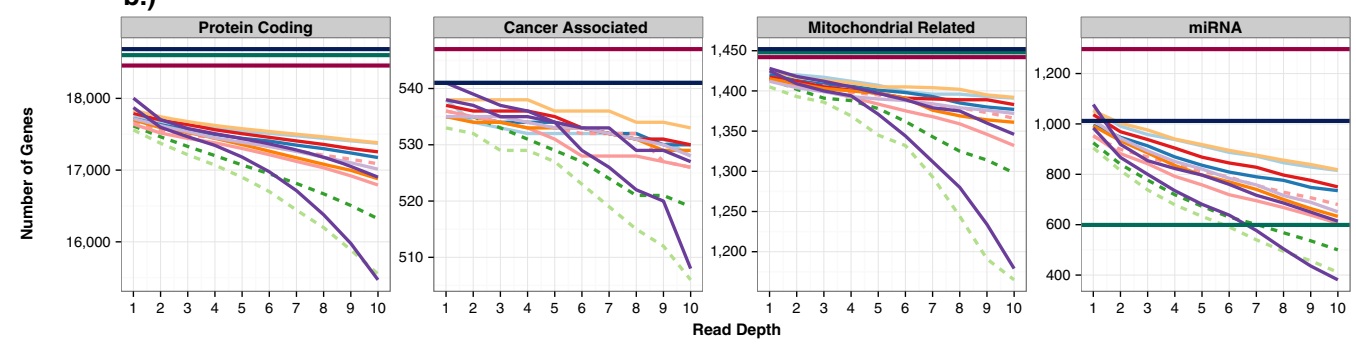

c.)

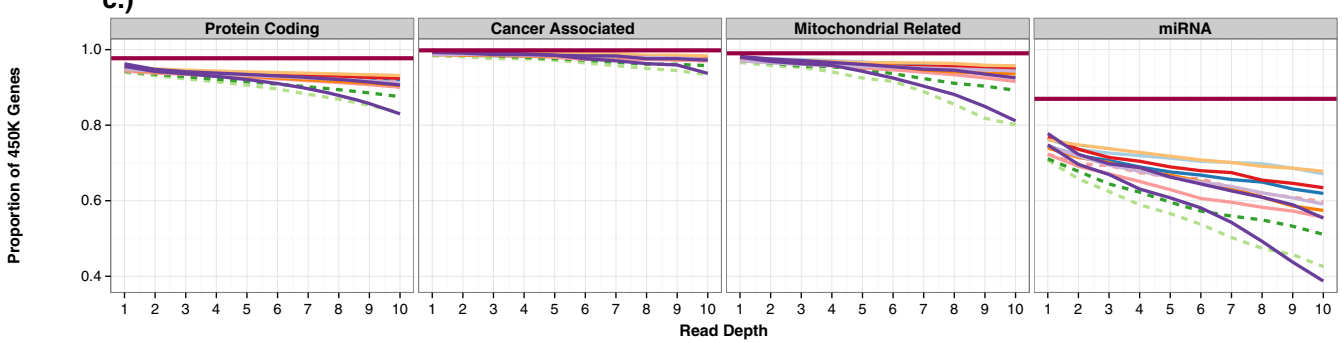

d.)

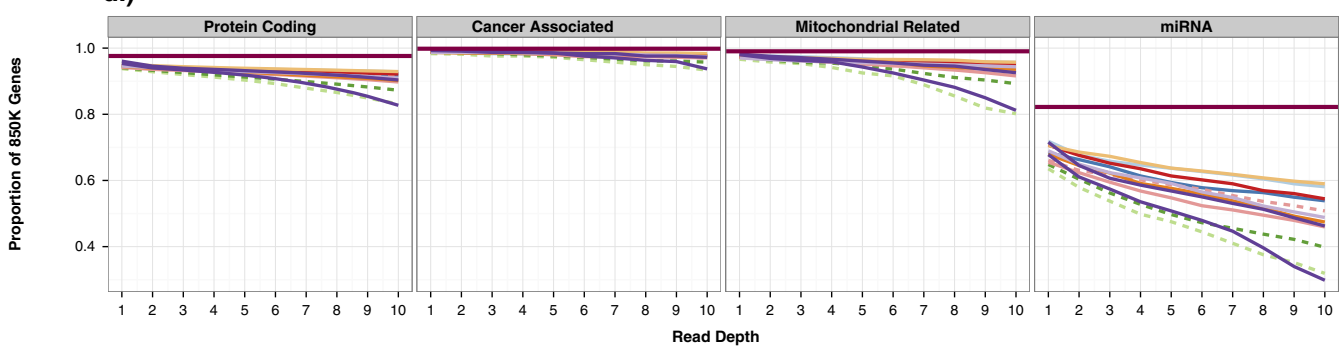

e.)

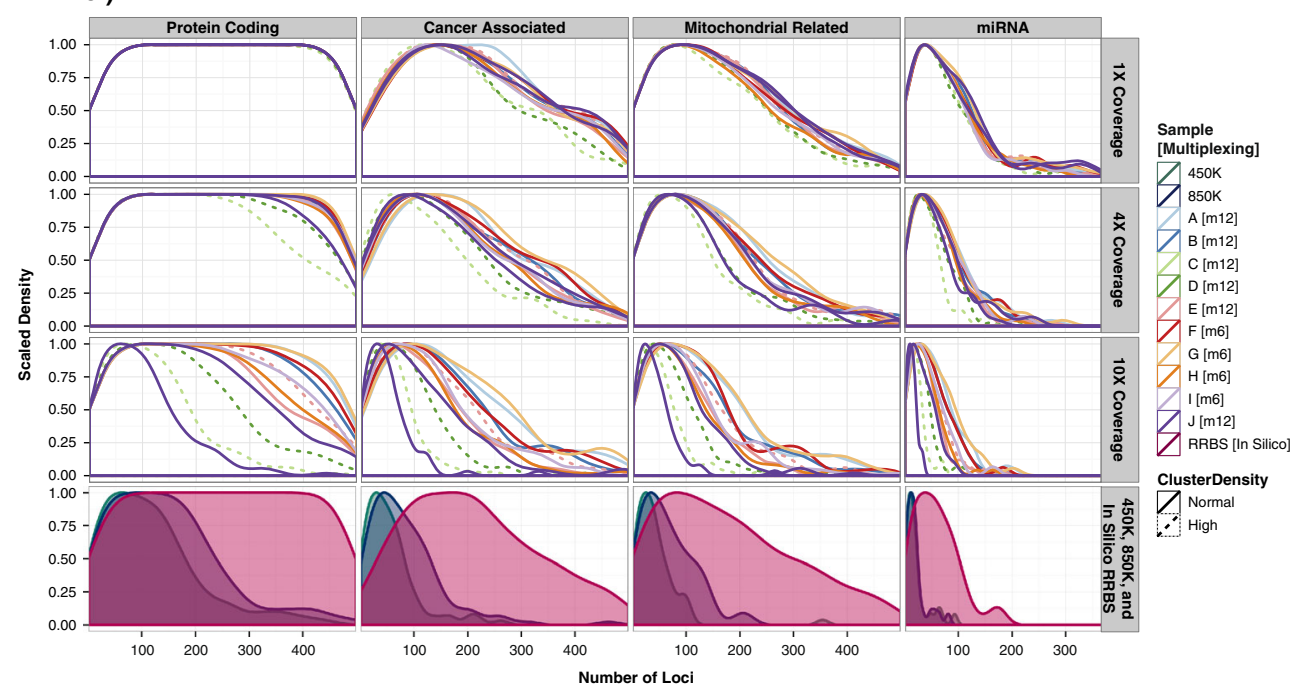


Fig. 2 Genomic coverage of 12 rmRRBS libraries at different read depths stratified by four types of genes. a The total number of CpG loci covered $\mathbf{b}$ the number of genes in which at least one CpG locus is covered $\mathbf{c}$ the proportion of genes on the Infinium HumanMethyation450 (450K) array for which at least once CpG locus is covered, and $\mathbf{d}$ the proportion of genes on the Infinium MethyationEPIC (850K) array for which at least once CpG locus is covered are plotted from $\geq 1 \times$ to $\geq 10 \times$ reads. In panels (a)-(d), the dark red horizontal lines indicate predicted RRBS genomic coverage, bioinformatically determined by performing RRBS in silico. In panels (a) and (b), the dark green horizontal lines indicate Infinium 450K genomic coverage, and the dark blue horizontal lines indicate Infinium $850 \mathrm{~K}$ genomic coverage. (e) The distribution of the number of loci measured in each gene that was covered is stratified in columns by type of gene and in rows by mRRBS read depth, with CpG density distributions for in silico RRBS, the Infinium 450K and the Infinium 850K plotted together in the fourth row. The peak of the density is the mode, and indicates most common number of CpG loci measured in each gene. In all panels, unique individuals A through J appear as different colored lines where solid lines indicate normal cluster density and dotted lines indicated high cluster density
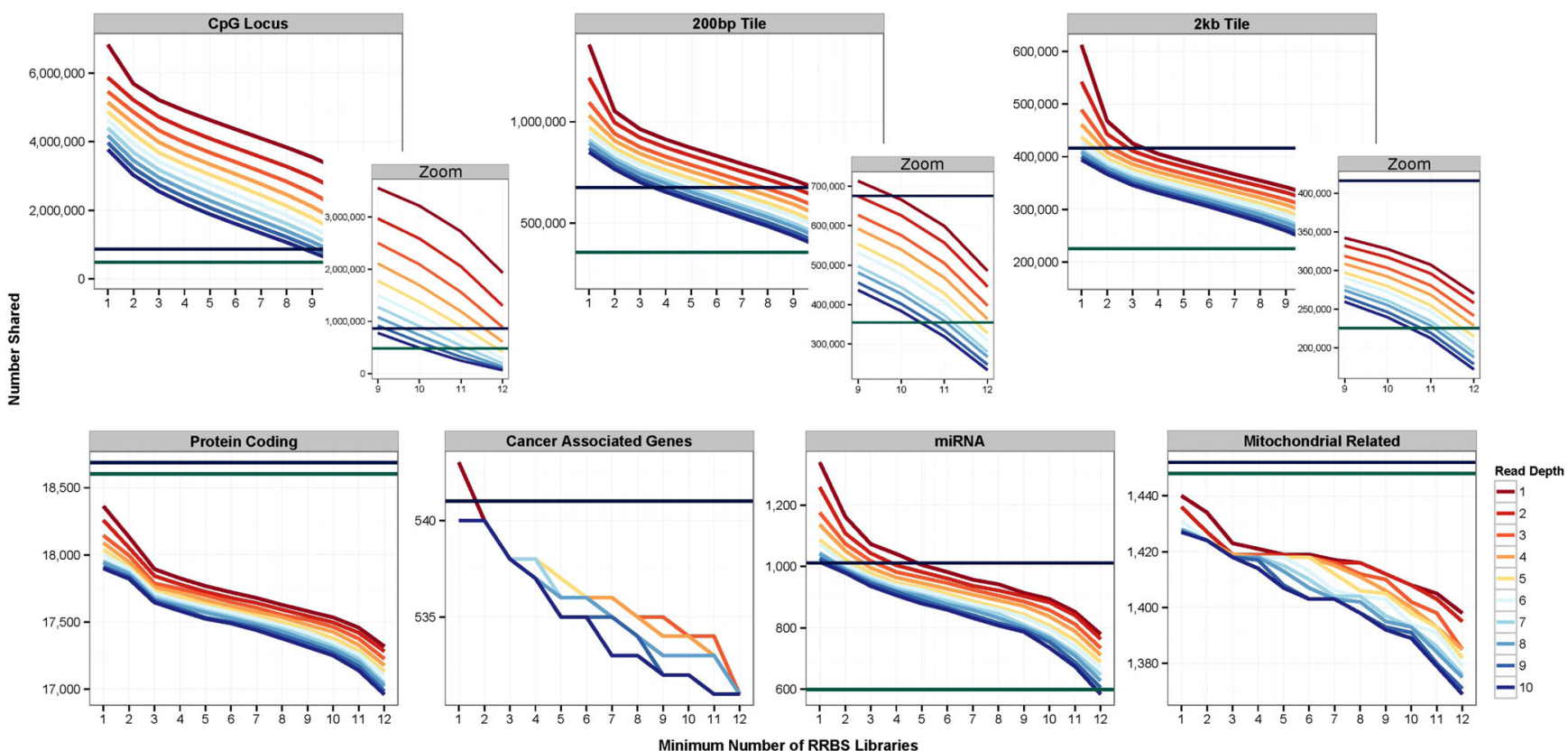

Fig. 3 Overlap in genomic coverage between different rmRRBS libraries. The number of CpG loci, CpG resort context-restricted genomic tiles up to 200 base pairs ( $200 \mathrm{bp}$ tiles) or 2000 base pairs ( $2 \mathrm{~kb}$ tiles) in length, protein coding genes, cancer-associated genes, microRNA genes, and nuclear encoded genes related to mitochondrial function are plotted relative to the number of rmRRBS libraries in which exact matches overlap. Different color lines correspond rmRRBS read depth, ranging from $\geq 1 \times$ to $\geq 10 \times$ reads per CpG locus, $200 \mathrm{bp}$, $2 \mathrm{~kb}$ tile, or gene. For reference, horizontal lines indicate the number of $\mathrm{CpG}$ loci, tiles or genes covered by the Infinium HumanMethylation450 (450K; dark green) and the Infinium MethyationEPIC (850K; dark blue)

related genes, and 30-68\% for microRNA genes (Fig. 2c-d). Regardless of genomic context, rmRRBS covered more CpG loci per region than the Infinium arrays (Figs. 1e, 2e). Likewise, rmRRBS measured a higher density of $\mathrm{CpG}$ loci in all 30 known human imprinting regions-which were covered at $\geq 10 \times$ by all 12 libraries-than the Infinium arrays (data not shown).

We next investigated overlap in genomic coverage between rmRRBS libraries, which is dependent on both read depth and the minimum number of libraries that must capture the same CpG locus. In addition to looking at individual CpG loci, we also divided the human genome into discrete genomic "tiles" that never span more than one type of $\mathrm{CpG}$ resort context. As expected, the correlation between DNAm at CpG loci decreased as the distance between the loci increased. However, the rate at which this correlation dissipated was dependent on the $\mathrm{CpG}$ resort context. DNAm in CpG islands was highly correlated across much larger regions than other resort contexts (Supplementary Fig. S1). To appraise the impact of this variability across regions on reproducibility, we created two sets of genomic tiles to increase overlap across libraries: one set with a maximum size of 200 basepairs (200 bp) and another set with a maximum size of 2000 basepairs $(2 \mathrm{~kb})$, the previously defined size of a $\mathrm{CpG}$ shore or shelf region. All 12 libraries at $\geq 4 \times$ read depth, and up to 10 libraries at $\geq 10 \times$, overlapped at more individual CpG loci $(>482,421), 200 \mathrm{bp}$ tiles $(>354,806)$ and $2 \mathrm{~kb}$ tiles $(>225,403)$ than are captured by the $450 \mathrm{~K}$ (Fig. 3). Furthermore, we found that different rmRRBS libraries covered a large number of exactly the same proteincoding, cancer-associated, microRNA and mitochondrial-related genes. The best overlap in coverage among the types of genes considered was in microRNAs. In fact, all 12 rmRRBS libraries at $\geq 4 \times$, and up to 10 libraries at $\geq 10 \times$, overlapped at $>715$ microRNA genes-more than the 599 microRNA genes present on the $450 \mathrm{~K}$. Figure 3 also displays putative $850 \mathrm{~K}$ coverage, which is particularly improved over the $450 \mathrm{~K}$ for $200 \mathrm{bp}$ tiles, $2 \mathrm{~kb}$ tiles and microRNA genes. The number of cancer-associated and mitochondrialrelated genes that overlapped between rmRRBS libraries were $\geq$ $95 \%$ and $\geq 90 \%$, respectively, of the number on the Infinium arrays.

\section{DNAm quantification}

In order to test the precision of rmRRBS DNAm measurements, and to determine the influence of experimental variables, we prepared 72 technical replicates from one individual's gDNA sample-12 libraries each under six different conditions, including two laboratory protocols (NEBNext and rmRRBS) with 60, 100, and $200 \mathrm{ng}$ of starting gDNA input (Supplementary Table S3). At 10x coverage and with $200 \mathrm{ng}$ of starting gDNA, the rmRRBS protocol 


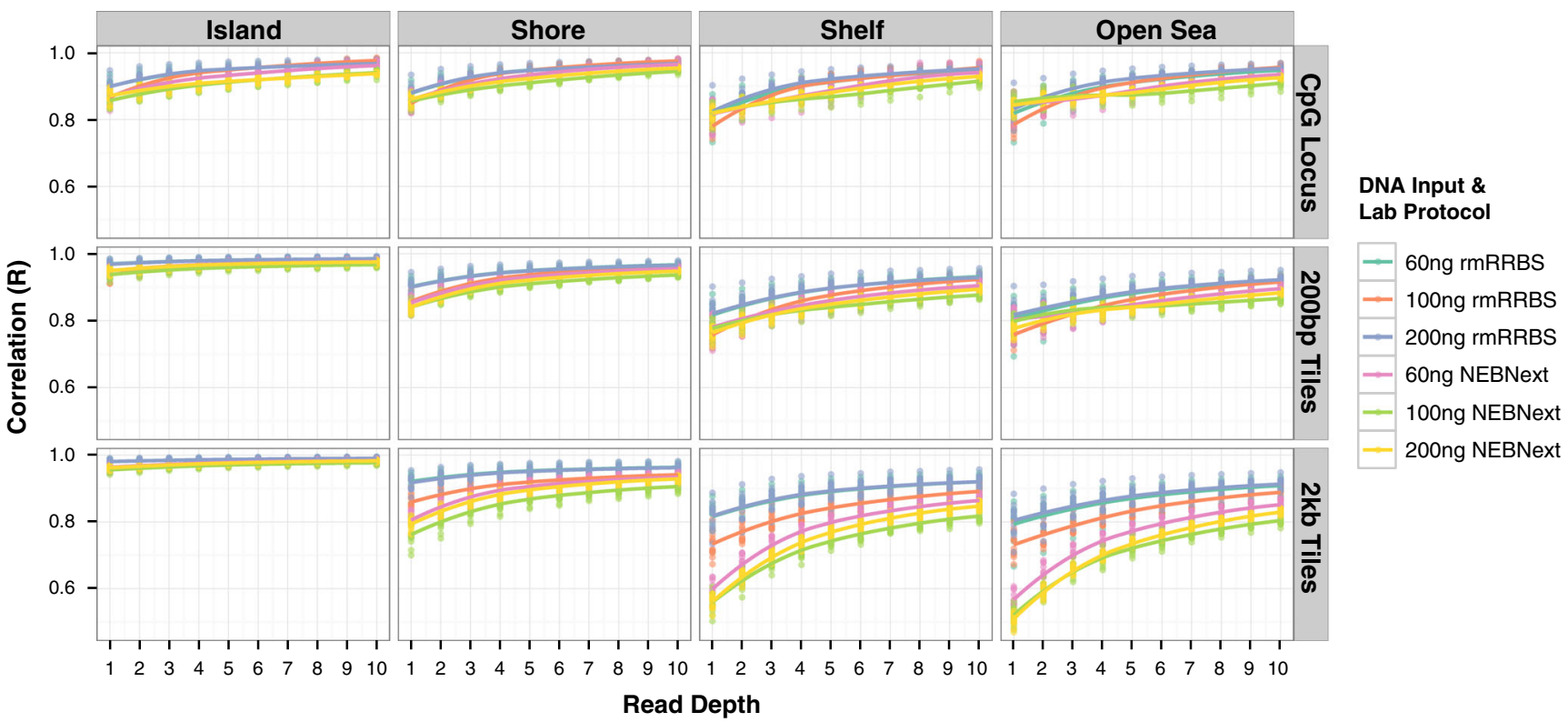

Fig. 4 Reproducibility of rmRRBS DNA methylation measurements. The Pearson correlations between DNA methylation measurements for combined reads from all 12 libraries prepared under each of six different experimental conditions and reads from the individual 12 libraries prepared under that condition, stratified by CpG resort context, ranging from $\geq 1 \times$ to $\geq 10 \times$ reads for the individual library, are plotted for CpG loci, 200 base pair genomic tiles ( $200 \mathrm{bp}$ tiles), and 2000 base pair genomic tiles ( $2 \mathrm{~kb}$ tiles)

captured a greater number of CpG loci on average, specifically among CpG islands, shores, and shelves on average. However, the NEBNext protocol tended to capture more open sea sites (Supplementary Table S4). For each CpG locus, 200 bp tile or 2 $\mathrm{kb}$ tile, we combined all sequencing reads for each condition to serve as standards. Stratifying by CpG context, we calculated the correlation of DNAm between each library and its standard, revealing that the reproducibility of rmRRBS DNAm measurements increased with read depth and CpG density (Fig. 4). At $\geq 10 x$ reads, R-values between libraries and their standards were $>0.9$ for islands and shores and $>0.85$ for shelves and open sea. Within CpG islands, reproducibility further improved when $200 \mathrm{bp}$ and $2 \mathrm{~kb}$ tiles were employed. Outside of CpG islands, using $200 \mathrm{bp}$ tiles had little effect, whereas using $2 \mathrm{~kb}$ tiles reduced reproducibility, particularly in shelves and open sea regions. Comparing DNAm measurements via rmRRBS to averaged triplicate Infinium measurements for the same gDNA sample, concordance between rmRRBS and Infinium DNAm measurements increased with both CpG density and rmRRBS read depth (Fig. 5). The Pearson correlation between rmRRBS estimated percent methylation and $450 \mathrm{~K}$ methylation level for all overlapping loci at $\geq 1 \mathrm{x}$ and $\geq 10 \mathrm{x}$ can be viewed in Genome Browser (Supplemental Materials). For ten libraries at $\geq 10 x$ reads, correlations with Infinium DNAm at individual CpG loci were $>0.95,>0.93,>0.85$, and $>0.88$ for islands, shores, shelves, and open sea, respectively. Using $200 \mathrm{bp}$ tiles rather than matching individual $\mathrm{CpG}$ loci increased the correlation between platforms, regardless of $\mathrm{CpG}$ context. Although using 2 $\mathrm{kb}$ tiles increased the correlation between platforms for DNAm measured in CpG islands even more than using 200 bp tiles, it decreased the correlation between platforms outside of $\mathrm{CpG}$ islands.

To assess the ability for rmRRBS to detect SNPs and measure ASM, we plotted ASM within the imprinting control region of $\mathrm{H} 19$ in proximity to a common G/T SNP ( $r$ 10840167) for which seven rmRRBS libraries were heterozygous with $\geq 4 x$ reads per allele. Epigenomic Roadmap data for the region with the SNP predicts leukocyte regulatory functions, including enhancer activity for lymphoid immune cells and repressed polycomb activity for myeloid immune cells. ${ }^{40}$ Across the five CpG loci captured on the same read as the SNP, we found that one allele was fully methylated and the other was fully unmethylated, suggesting that these loci exhibit parental imprinting (Fig. 6). Since the Infinium array cannot measure ASM, the expected methylation value for these loci is $50 \%$ (the average of DNAm from the two alleles). Instead the two samples that showed higher methylation on the T-allele had high ( 89\%) methylation at the locus that overlapped with this region on the Infinium $450 \mathrm{~K}$, whereas the methylation level ranged from 35 to $48 \%$ for the rest of the samples.

\section{DISCUSSION}

We empirically assessed a derivative of RRBS optimized for highthroughput, called rmRRBS, and Infinium BeadChip technology. Our work suggests that there are many trade-offs to consider when selecting between array-based and/or sequencing-based DNAm platforms for human research studies. Compared to the Infinium array, NGS-based rmRRBS is capable of covering more CpG loci and a greater abundance of CpG shores, which are thought to be important for exposure-related epigenetic modifications, as well as open sea regions. Additionally, rmRRBS tends to cover more CpG loci within a given region than the Infinium. Unlike array-based approaches, however, the exact same CpG loci are not always measured across rmRRBS libraries. Moreover, the number of reads covering each site impacts quantitative estimation of DNAm, thereby influencing reproducibility. All rmRRBS libraries are generated by enzymatic digestion of the genome, so there is no guarantee that a fragment will be generated even if the correct restriction sites flank the area. Furthermore, even if the digestion generates a particular fragment, it must also be properly ligated onto adapters, bisulfite converted, PCR amplified, sequenced, and successfully aligned in order for a CpG locus therein to be analyzed as a discrete read. Thus, there will usually be some experimental variation between libraries, which manifests as differences in genomic coverage and depth. This work considered variability due to DNA input quantity for a commonly used biologic sample: human blood. Future work should explore other potential sources of variability, particularly those affecting DNA quality, such as different origins (e.g., tumor 


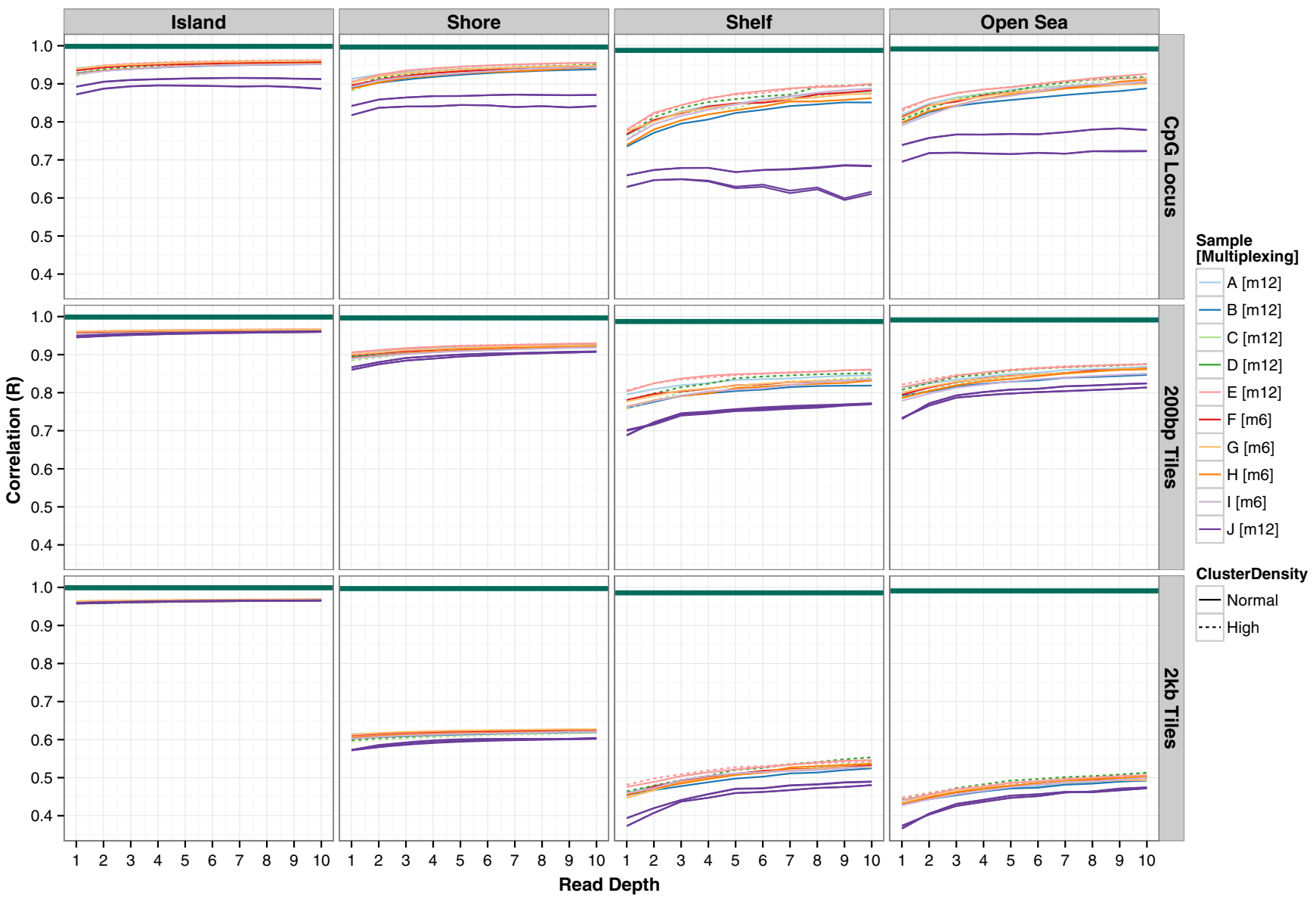

Fig. 5 Concordance of rmRRBS and Infinium DNA methylation measurements. The Pearson correlations between DNA methylation measurements from 12 rmRRBS libraries derived from ten different participants' (labeled A-J) gDNA and DNA methylation measurements for the corresponding Infinium HumanMethylation450 (450K) data derived from the same ten participants' gDNA is plotted from $\geq 1 \times$ to $\geq 10 \times$ rmRRBS reads. Results are stratified by CpG resort context and shown for CpG loci, 200 base pair genomic tiles (200 bp tiles), and 2000 base pair genomic tiles ( $2 \mathrm{~kb}$ tiles)

tissue, cell lines, cultured primary cells), storage conditions, purities, and extraction methods. Since RRBS libraries are generated by fractionating DNA early in the procedure, premature fractionation is unlikely to significantly affect results. This suggests that rmRRBS is a suitable platform for samples of uncertain or poorer quality, such as DNA derived from formalin fixed paraffin embedded tissue and clinical samples that were processed or stored under sub-optimal conditions. However, additional systematic experiments are needed to assess rmRRBS performance relative to array-based approaches for poorer quality DNA.

Considering DNAm across tiles, rather than individual CpG loci, can improve overlap across rmRRBS libraries. While different libraries may not cover precisely the same CpG loci, they may cover CpG loci in close proximity. As anticipated, the impact of tile size on rmRRBS reproducibility and concordance with Infinium measurements depended on the variability of DNAm within the genomic region. For less variable elements, such as CpG islands, even relatively large tiles improved the precision of DNAm estimates. In regions where the correlation between loci dropped quickly with distance, such as shores and shelves, smaller tiles were necessary. Our capacity to estimate these regional DNAm patterns is facilitated by the greater density of CpG loci among contexts covered compared to the Infinium.

Unlike Infinium BeadChip arrays, rmRRBS allows for both genotyping (i.e., SNP detection) and the measurement of ASM, making it a powerful platform for studies of imprinting and other types of monoallelic DNAm. Our examination of the $11 \mathrm{p} 15$ imprinting region (specifically $H 19$ ) not only revealed that rmRRBS is effective at measuring ASM, but it also confirmed that Infinium DNAm quantification can be confounded by the status of SNPs near the target $\mathrm{CpG}$, thus introducing bias that is usually avoided by removing all known SNP proximal Infinium probes from analysis.

If sample material or DNA is scarce, it may not be possible to use Infinium BeadChip arrays, which require $500 \mathrm{ng}-1 \mu \mathrm{g}$ of DNA (or WGBS, which requires $3 \mu \mathrm{g}$ of DNA). Herein, we demonstrate that rmRRBS can measure genome-wide DNAm using 60-200 ng of DNA. Both platforms require high-quality DNA input. There is a possibility that rmRRBS may be more sensitive to microbial contamination, such as might be found in saliva or buccal cell DNA, than the Infinium array, but this remains to be tested. The overall costs of running rmRRBS and the Infinium are comparable considering requisite labor, reagents, and bioinformatics. If the investigator has abundant DNA, Infinium arrays are likely the better option due to their consistency in both genomic coverage and DNAm estimates. There are some concerns involving dye-bias between the different probe types, as well as positional and batch effects on Infinium arrays, but these have been mostly resolved in well-established bioinformatics pipelines. ${ }^{22,24,41,42}$ On the other hand, rmRRBS offers greater flexibility in the genomic regions that are detected, with the potential to investigate DNAm at sites that are not interrogated by Infinium arrays, which could lead to the discovery of novel biomarkers that would be missed otherwise. In our work, we found that a larger number (and a different pool) of microRNA genes were covered by rmRRBS than the Infinium 450K. 

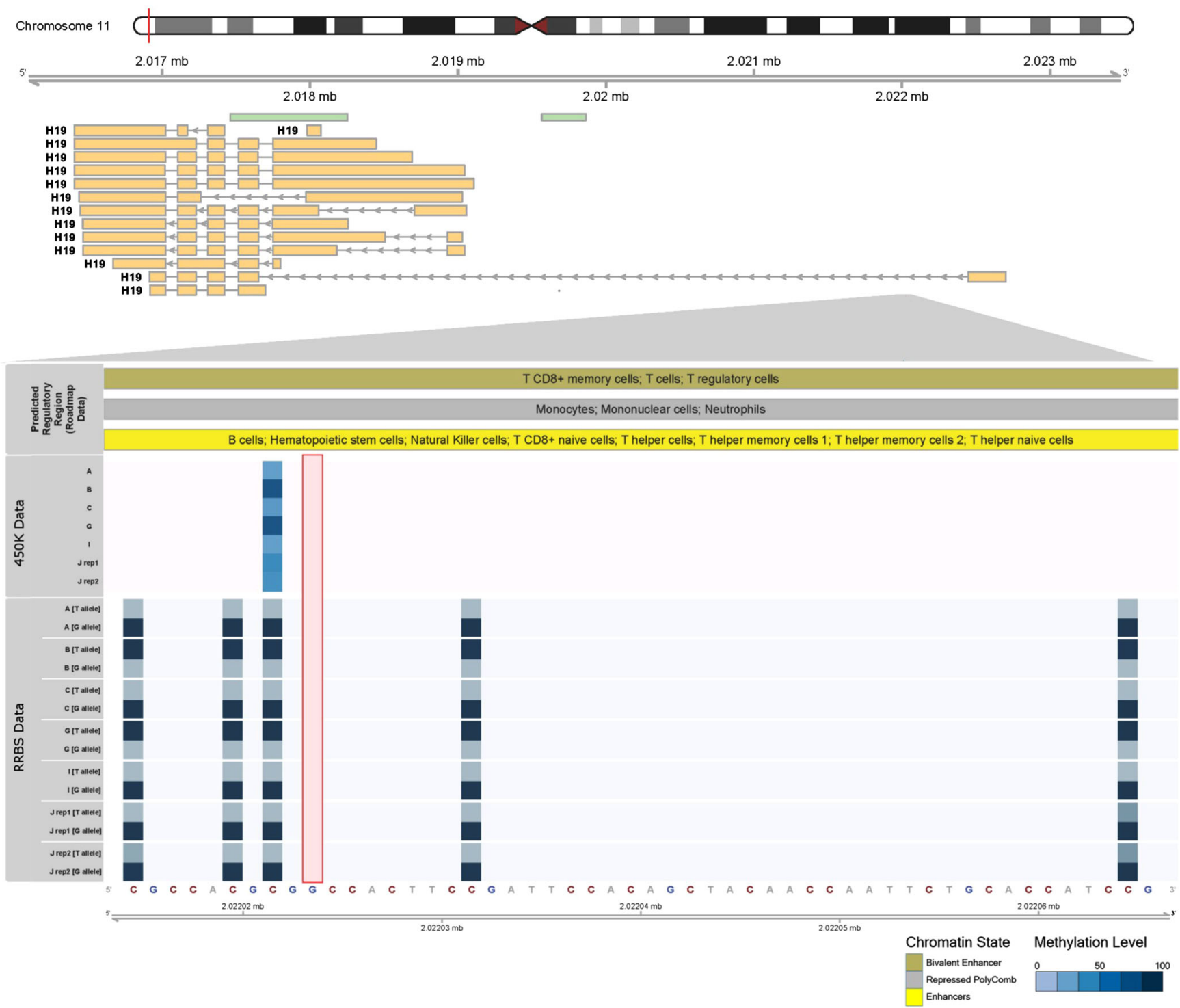

Fig. 6 Genotype and allele-specific DNA methylation are measured by rmRRBS but not the Infinium BeadChip. A diagram illustrating the position of the target region in $H 19$ within the 11 p15 imprinting region is shown at the top of the figure. The primary sequencing of DNA nucleotides appears at the bottom, with a G/T single nucleotide polymorphism (SNP) highlighted in a red box in the body of the figure. Monoallelic DNA methylation for the five CpG loci covered by rmRRBS, as well as overall DNA methylation for the single CpG locus covered by the Infinium HumanMethylation450 (450K) array, are shown for the six individuals (including one replicate of J) who were heterozygous for the G/T SNP with blue saturation of heatmap cells indicating DNA methylation levels. Predicted regulatory functions based on Epigenetic Roadmap chromatin state data is shown above the DNA methylation heatmaps

This suggests that rmRRBS is of particular utility for researchers interested in studying DNAm in human microRNA genes. Furthermore, epigeneticists interested in SNP genotyping and/or allele-specific DNAm would benefit from employing rmRRBS in their research, and the method has the potential for clinical applications including diagnostics for imprinting disorders and the detection of diagnostic and prognostic markers, as well as identification of therapeutic targets. ${ }^{43}$

It is likely that experimental and analytic approaches to RRBS derivatives will be further refined in the near future. In order to decrease variability in coverage and increase both read depth and overlap between libraries, researchers may select a narrower range of fragments to enrich for certain CpG contexts, and/or multiplex fewer samples per lane to return more reads per library. Experiments that systematically explore fragment selection and multiplexing would help elucidate these possibilities.
Improvements to genomic tiling and other regional-based analyses could also augment our ability to detect differences between samples, such as those associated with diseases or environmental exposures. Moreover, regional changes in DNAm associated with a variable interest may have more functional relevance than locus-specific changes, since gene expression is not always correlated with DNAm at individual CpG sites. Analysis pipelines that take advantage of the large number of overlapping genomic regions between any given two RRBS libraries could yield more powerful results; perhaps we can even adapt analytic approaches that have already been developed in other fields.

\section{METHODS}

Details available as Supplement. 
Microarray data

Microarray data will be posted to GEO in accordance with MIAME.

\section{ACKNOWLEDGMENTS}

We would like to thank Richard Thomas Barfield and Pia Pafundi for help with preliminary experiments, and Alexander Meissner for expert advice. J.J. Carmona was supported by Ruth L. Kirschstein National Research Service Award (NRSA) for Individual Postdoctoral Fellows F32ES024068 from the National Institute of Environmental Health Science, National Institutes of Health, and by Training Grant T32ES007069 in Environmental Epidemiology from the National Institute of Environmental Health Science, National Institutes of Health. W.P. Accomando Jr. was supported by Training Grant T32CA009001 in Cancer Epidemiology from the National Cancer Institute, National Institutes of Health. A.M. Binder was supported by Training Grant T32HD060454 in Reproductive, Perinatal and Pediatric Epidemiology from the National Institute of Child Health and Human Development, National Institutes of Health. This work was also supported by grant award number R01ES021733 and R01ES015172 from the National Institute of Environmental Health Science, National Institutes of Health, and by grant award number R01CA158313 from the National Cancer Institute, National Institutes of Health. The content is solely the responsibility of the authors and does not necessarily represent the official views of the National Institutes of Health. The VA Normative Aging Study is supported by the Cooperative Studies Program Epidemiology Research and Information Center of the US. Department of Veterans Affairs and is a component of the Massachusetts Epidemiology Research and Information Center, Boston, MA. The Translational Genomics Core at Partners Personalized Medicine.performed the Illumina HiSeq2500 sequencing and the Infinium HumanMethylation450 BeadChip array processing and scanning (lllumina iScan) reported in this work. This work was supported by grants from the National Institutes of Health: R01ES021733, R01ES015172, R01CA158313, T32CA009001, T32ES007069, T32HD060454, and F32ES024068.

\section{AUTHOR CONTRIBUTIONS}

J.J.C., W.P.A., and A.M.B. contributed equally to this work as first authors. J.J.C and W.P. A. conceived of the study, designed the experiments, coordinated the work, performed experiments, and wrote the paper. A.M.B. conceived of the study, participated in study design and coordination, analyzed the data, generated figures, and wrote the paper. J.N.H. and L.P. designed and performed bioinformatic analyses. B.I., X.L., and P.S.V. participated in study design and performed experiments. A.C.J. participated in study design and analysis. J.S. participated in study design and coordination. S.S.A. participated in study design, coordination, and core laboratory experiments. A.A.B. and K.B.M. conceived of the study, participated in study design, helped coordinate the study, and supervised all aspects of the work.

\section{COMPETING INTERESTS}

The authors declare no competing interests.

\section{REFERENCES}

1. Berger, S. L., Kouzarides, T., Shiekhattar, R. \& Shilatifard, A. An operational definition of epigenetics. Genes. Dev. 23, 781-783 (2009).

2. Khavari, D. A., Sen, G. L. \& Rinn, J. L. DNA methylation and epigenetic control of cellular differentiation. Cell Cycle 9, 3910-3913 (2010).

3. Zaidi, S. K. et al. Bookmarking the Genome: maintenance of epigenetic information. J. Biol. Chem. 286, 18355-18361 (2011).

4. Bird, A. DNA methylation patterns and epigenetic memory. Genes Dev. 16, 6-21 (2002).

5. Richmond, T. J. \& Davey, C. A. The structure of DNA in the nucleosome core Nature 423, 145-150 (2003)

6. Lande-Diner, L. \& Cedar, H. Silence of the genes-mechanisms of long-term repression. Nat. Rev. Genet. 6, 648-654 (2005).

7. Shi, H., Wang, M. X. \& Caldwell, C. W. CpG islands: their potential as biomarkers for cancer. Exp. Rev. Mol. Diagn. 7, 519-531 (2007).

8. Sandoval, J. et al. Validation of a DNA methylation microarray for 450,000 CpG sites in the human genome. Epigenetics. 6, 692-702 (2011).

9. Ji, $\mathrm{H}$. et al. A comprehensive methylome map of lineage commitment from hematopoietic progenitors. Nature 467, 338-342 (2010).

10. Accomando, W. P., Wiencke, J. K., Houseman, E. A., Nelson, H. H. \& Kelsey, K. T. Quantitative reconstruction of leukocyte subsets using DNA methylation. Genome Biol. 15, R50 (2014).

11. Houseman, E. A. et al. DNA methylation arrays as surrogate measures of cell mixture distribution. BMC Bioinf. 13, 86 (2012)
12. Reinius, L. E. et al. Differential DNA methylation in purified human blood cells: implications for cell lineage and studies on disease susceptibility. PLOS ONE 7 e41361 (2012).

13. Jaffe, A. E. \& Irizarry, R. A. Accounting for cellular heterogeneity is critical in epigenome-wide association studies. Genome Biol. 15, R31 (2014).

14. Doi, A. et al. Differential methylation of tissue- and cancer-specific CpG island shores distinguishes human induced pluripotent stem cells, embryonic stem cells and fibroblasts. Nat. Genet. 41, 1350-1353 (2009).

15. Hansen, K. D. et al. Increased methylation variation in epigenetic domains across cancer types. Nat. Genet. 43, 768-775 (2011).

16. Irizarry, R. A. et al. The human colon cancer methylome shows similar hypo- and hypermethylation at conserved tissue-specific CpG island shores. Nat. Genet. 41, 178-186 (2009).

17. Ulahannan, N. \& Greally, J. M. Genome-wide assays that identify and quantify modified cytosines in human disease studies. Epigenet. Chromat. 8, 5 (2015).

18. Dedeurwaerder, S. et al. Evaluation of the Infinium Methylation $450 \mathrm{~K}$ technology. Epigenomics 3, 771-784 (2011).

19. Bibikova, M. et al. High density DNA methylation array with single CpG site resolution. Genomics 98, 288-295 (2011).

20. Bibikova, M. et al. Genome-wide DNA methylation profiling using Infinium ${ }^{\odot}$ assay. Epigenomics 1, 177-200 (2009).

21. Bibikova, M. et al. High-throughput DNA methylation profiling using universal bead arrays. Genome Res. 16, 383-393 (2006).

22. Aryee, M. J. et al. Minfi: a flexible and comprehensive Bioconductor package for the analysis of Infinium DNA methylation microarrays. Bioinformatics. 30, 1363-1369 (2014).

23. Dedeurwaerder, S. et al. A comprehensive overview of Infinium HumanMethylation450 data processing. Brief Bioinform. 15, 929-941 (2014).

24. Assenov, Y. et al. Comprehensive analysis of DNA methylation data with RnBeads. Nat. Methods. 11, 1138-1140 (2014).

25. Price, M. E. et al. Additional annotation enhances potential for biologicallyrelevant analysis of the Illumina Infinium HumanMethylation450 BeadChip array. Epigenet. Chromat. 6, 4 (2013).

26. Zhang, X., Mu, W. \& Zhang, W. On the analysis of the Illumina 450 k array data: probes ambiguously mapped to the human genome. Front. Genet. 3, 73 (2012).

27. Ziller, M. J., Hansen, K. D., Meissner, A. \& Aryee, M. J. Coverage recommendations for methylation analysis by whole-genome bisulfite sequencing. Nat. Methods, doi:10.1038/nmeth.3152 (2014).

28. Xi, Y. \& Li, W. BSMAP: whole genome bisulfite sequence MAPping program. BMC Bioinf. 10, 232 (2009)

29. Boyle, P. et al. Gel-free multiplexed reduced representation bisulfite sequencing for large-scale DNA methylation profiling. Genome Biol. 13, R92 (2012).

30. Gu, H. et al. Preparation of reduced representation bisulfite sequencing libraries for genome-scale DNA methylation profiling. Nat. Protoc. 6, 468-481 (2011).

31. Garrett-Bakelman, F. E. et al. Enhanced reduced representation bisulfite sequencing for assessment of DNA methylation at base pair resolution. J. Vis. Exp. JoVE, doi:10.3791/52246 (2015).

32. Guo, H. et al. Single-cell methylome landscapes of mouse embryonic stem cells and early embryos analyzed using reduced representation bisulfite sequencing Genome Res. 23, 2126-2135 (2013).

33. Martin-Trujillo, A. et al. Genotype of an individual single nucleotide polymorphism regulates DNA methylation at the TRPC3 alternative promoter. Epigenetics. 6 , 1236-1241 (2011).

34. Lin, P.-C. et al. Epigenomic alterations in localized and advanced prostate cancer. Neoplasia N. Y. N 15, 373-383 (2013).

35. Gertz, J. et al. Analysis of DNA methylation in a three-generation family reveals widespread genetic influence on epigenetic regulation. PLoS Genet. 7, e1002228 (2011).

36. Harris, R. A. et al. Comparison of sequencing-based methods to profile DNA methylation and identification of monoallelic epigenetic modifications. Nat Biotechnol. 28, 1097-1105 (2010).

37. Ratajczak, M. Z. Igf2-H19, an imprinted tandem gene, is an important regulator of embryonic development, a guardian of proliferation of adult pluripotent stem cells, a regulator of longevity, and a 'passkey' to cancerogenesis. Folia Histochem. Cytobiol. Pol. Acad. Sci. Pol. Histochem. Cytochem. Soc 50, 171-179 (2012).

38. Sparago, A. et al. Mechanisms causing imprinting defects in familial BeckwithWiedemann syndrome with Wilms' tumour. Hum. Mol. Genet. 16, 254-264 (2007).

39. Scelfo, R. A. M. et al. Loss of methylation at chromosome $11 \mathrm{p} 15.5$ is common in human adult tumors. Oncogene. 21, 2564-2572 (2002).

40. Roadmap Epigenomics Consortium et al. Integrative analysis of 111 reference human epigenomes. Nature 518, 317-330 (2015).

41. Pidsley, R. et al. A data-driven approach to preprocessing Illumina $450 \mathrm{~K}$ methylation array data. BMC Genomics. 14, 293 (2013).

42. $\mathrm{Wu}, \mathrm{M}$. C. et al. A systematic assessment of normalization approaches for the Infinium 450K methylation platform. Epigenetics 9, 318-329 (2014). 
43. Heyn, H. \& Esteller, M. DNA methylation profiling in the clinic: applications and challenges. Nat. Rev. Genet. 13, 679-692 (2012).

c) (i) This work is licensed under a Creative Commons Attribution 4.0 International License. The images or other third party material in this article are included in the article's Creative Commons license, unless indicated otherwise in the credit line; if the material is not included under the Creative Commons license, users will need to obtain permission from the license holder to reproduce the material. To view a copy of this license, visit http://creativecommons.org/licenses/by/ $4.0 /$

(c) The Author(s) 2017

Supplementary Information accompanies the paper on the npj Genomic Medicine website (doi:10.1038/s41525-017-0012-9). 